\title{
Retrograde temporal order amnesia resulting from damage to the fornix
}

Fumihiko Yasuno, Masayuki Hirata, Hiroshi Takimoto, Masaaki Taniguchi, Yoshitsugu Nakagawa, Yoshitaka Ikejiri, Takashi Nishikawa, Kazuhito Shinozaki, Hirotaka Tanabe, Yoshiro Sugita, Masatoshi Takeda

\begin{abstract}
Department of Neuropsychiatry F Yasuno

Y Nakagawa

Y Ikejiri

T Nishikawa

K Shinozaki

M Takeda

Department of Neurosurgery, Osaka University Medical School, Suita, Osaka, Japan

M Hirata

M Taniguchi

Medical Science for Health, 3rd Division, Faculty of Health and

Sport Sciences, Osaka University, Suita, Osaka, Japan

F Yasuno

Y Sugita for remotely acquired material is described, after a lesion including the bilateral anterior fornix and adjacent anterior thalamus. The patient displayed a deficit in the temporal order tasks for remotely acquired information. Neither frontal cognitive deficits nor recognition deficits can account for this patient's poor temporal memory. This retrograde temporal order memory impairment without content memory deficits were not seen in previously reported thalamic amnesic patients. Accordingly, the present patient's poor retrograde temporal memory could hardly be explained by only a thalamic lesion. It is concluded that the patient's impairment of temporal order memory for the retrograde material is probably due to the direct disconnection between the frontal lobe and the hippocampus by disruption of the fornix.
\end{abstract}

Department of Neurosurgery, Suita Municipal Hospital, Suita, Osaka, Japan H Takimoto

Department of Neuropsychiatry, Ehime University School of Medicine, Ehime, Japan

H Tanabe

Correspondence to: Dr Fumihiko Yasuno, Department of

Neuropsychiatry, Osaka

University Medical School,

2-2 Yamadaoka, Suita, 565,

Japan. Telephone 00816879

3058; fax 008168793059 .

Received 13 March 1998 and in final form

10 December 1998

Accepted 22 December 1998
Abstract

Some amnesic patients show an impairment of temporal order memory that cannot be accounted for by content memory deficits. The performance of an amnesic patient on memory tasks assessing the patient's content and temporal memories

(F Neurol Neurosurg Psychiatry 1999;67:102-105)

Keywords: fornix; thalamus; amnesia; temporal order

Previous studies have indicated that some amnesic patients have an impairment of temporal order memory, and it was suggested that different brain structures such as the limbic-diencephalic and frontal regions may contribute to the process that underlies temporal memory, but each may do so in different ways. ${ }^{1}$ The fornix is known to connect these temporal memory related regions, ${ }^{2-4}$ and there are several reports in the literature on memory disorders after fornix lesions in humans. ${ }^{5-7}$ However, the effect of a fornix lesion on temporal order memory has not yet been elucidated. Here we report on a patient who developed retrograde temporal order amnesia after a lesion including the bilateral fornix.

\section{Method}

CASE REPORT

A 51 year old right handed high school educated woman was admitted to our hospital in November 1995, after an episode of disorientation, amnesia, headache, nausea, and vomiting. Brain CT and MRI disclosed a cavernous haemangioma situated in the anterior part of the third ventricle with obstructive hydrocephalus. A fenestration of transparent septum and placement of a ventriculoperitoneal shunt for the hydrocephalus were performed. The tumour was not resected, to prevent hemorrhage. There were no operative complications and the patient's recovery in the immediate postoperative period was uneventful.

Several weeks after the operation, she was unable to recall any of the events surrounding her illness, and showed difficulty in recent memory retrieval. Brain MRI performed 2 months after the surgery showed an area of cavernous haemangioma in the anterior part of the third ventricle (figure A). The lesion involved the bilateral anterior fornix and the anterior parts of medial thalamus (figure B, C). Single photon emission computed tomography (SPECT) carried out after the surgery showed an abnormal distribution of the tracer in the lesion, whereas in other areas its uptake was normal.

\section{Neuropsychological evaluation}

An extensive neuropsychological evaluation was performed between 2 and 5 months after the operation. The patient's scores are given in the table. There were no remarkable abnormalities except for anterograde memory deficits. The patient's retrograde memory was assessed by a structured interview covering past personal events. In addition, the famous faces and events test was administered, on which she was shown pictures and asked the names of the pictured subjects or events. In both of these tests, there was no evidence of significant retrograde amnesia.

\section{Retrograde temporal order memory}

When we asked the patient about the temporal order of the past events, she showed difficulties in both the autobiographical and the famous persons or events tests. For example, she could 
tell us the details of travels with her family in the previous year and 5 years earlier, but when we asked her which event had occurred first or after the other, she made a mistake. She knew very well that a major earthquake had occurred in Japan in the previous year and that a major international airport was opened nearby 2 years earlier, both of which were very familiar events in her living area, but she could not decide which event had occurred first or second. In the temporal order tasks, she stated that she felt confused when remembering the order of the events, even though the events were familiar to her.

\section{Experimental procedures for testing retrograde temporal order memory}

Retrograde temporal order memory was evaluated using public events arrangement tasks from Bowers et $a l^{18}$ For this task, items were taken from the pictures used in the famous faces or events test for the evaluation of the patient's past event memory. A total of 40 items was used, consisting of 10 from each decade from the 1960s to the 1990s. Each item was presented on a card. For each trial, the subject was presented with a set of four cards, one for each decade, arranged in a random order. The subject was then asked to order the four events according to their time of occurrence, starting with the most remote event and completing the set with the most recent one. Ten trials were administered in this way. The accuracy of placement per decade and global arrangement score were calculated. The accuracy of placement per decade was based on the number of items per decade placed in the correct position within a set. The global arrangement score was a vector score based on the distance between the correct response and the subject's response. For each item within a set, the absolute difference was taken between the position given to the item by the subject and its correct items within a set. For example, a response (1960s,

A

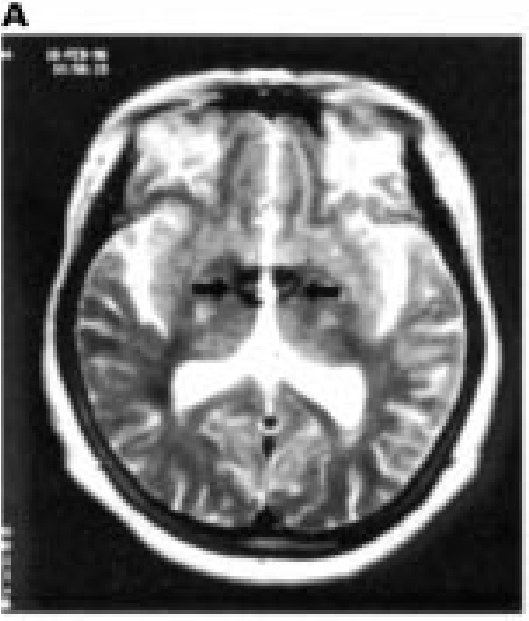

B
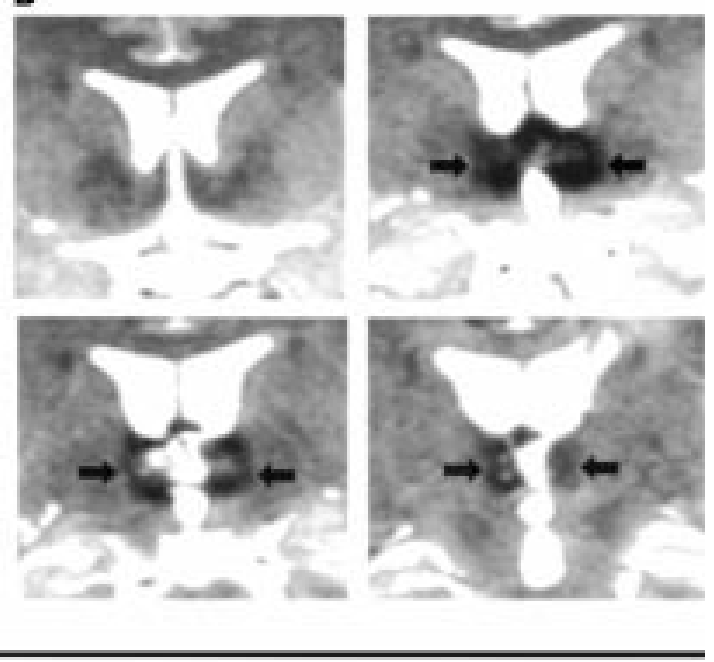

C
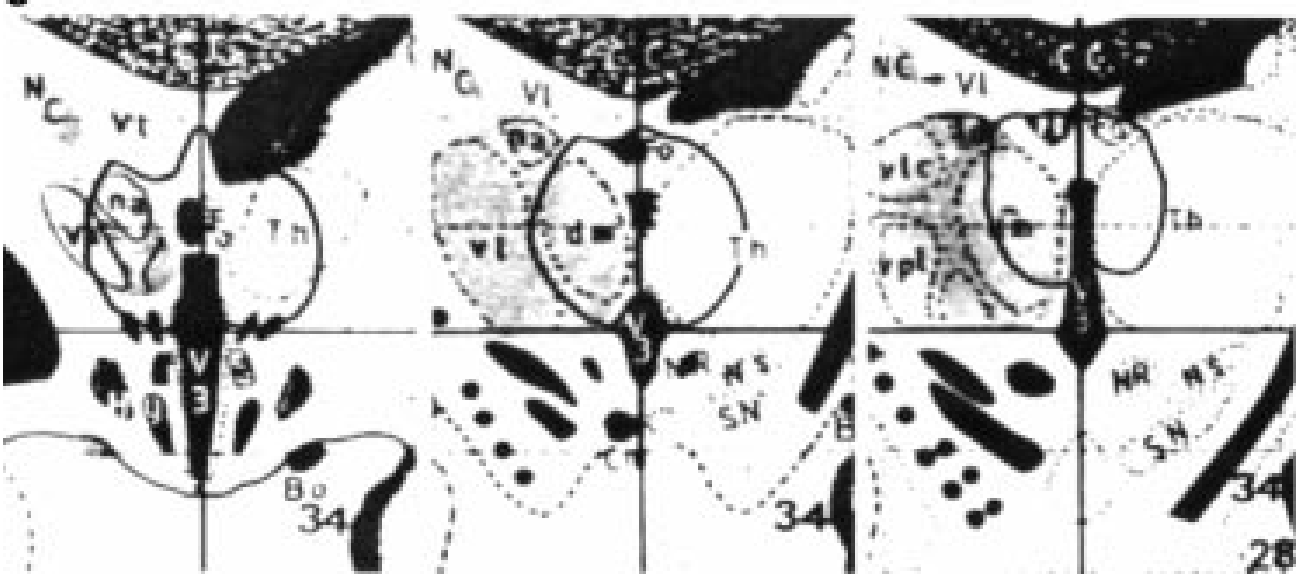

(A) Axial view of the T2 weighted MR images of the patient's brain. The arrows indicate the lesion of cavernous haemangioma. (B) MRI of the diencephalic region (phase T2). Four coronal sections are shown, the most anterior level on the left top, the most posterior level on the right bottom. The hypodense area indicated by the arrows shows the patient's lesion (for exact location see $(C)$ ). (C) Lesions plotted on the corresponding sections in the coronal planes (first plane $V C A=4 \mathrm{~mm}$, second plane $V C A=12 \mathrm{~mm}$, third plane $V C A=16 \mathrm{~mm}$ ) from the atlas of Talairach and Tournoux. ${ }^{8}$ The circled areas represent the patient's lesion. $B o=O p t i c$ tract; $C c=$ corpus callosum; $C m=$ mamillary body; Fo=fornix; $H y=$ hypothalamus $; c=$ caudate nucleus; $N r=$ red nucleus; $N s=$ subthalamic nucleus; $\operatorname{Sn}=$ substantia nigra; $T$ Th=thalamus; $n a=$ anterior nucleus; $d m=$ dorsomedial nucleus; va=ventral anterior nucleus; vl=ventral lateral nucleus; vpl=ventral posterior lateral nucleus; $V l=l a t e r a l$ ventricle; $V 3=$ third ventricle. 
3 Swanson LW, Cowan WM. An autoradiographic study of the organization of the efferent connections of the hippoc-

ampal formation in the rat. $\mathcal{F}$ Comp Neurol $1979 ; 172: 49-84$.
4 van Hosen GW. Neural systems of the non-human primate forebrain implicated in memory. Ann N Y Acad Sci 1985;444:97-112.

5 Hodges JR, Carpentar K. Anterograde amnesia with fornix damage following removal of IIIrd ventricle colloid cyst. $\mathcal{F}$ Neurol Neurosurg Psychiatry 1991;54:633-8.

6 McMackin D, Cockburn J, Anslow P, et al. Correlation of fornix damage with memory impairment in six case of colloid cyst removal. Acta Neurochir 1995;135:12-8.

7 Calabrese P, Markowitsch HJ, Hardes AG, et al. Fornix damage and memory. A case report. Cortex 1995;31:55564.

8 Talairach J, Tournoux P. Co-planar stereotaxic atlas of the brain. New York: Thieme, 1988.

9 de Renzi E, Faglioli P. Normative data and screening power of a shortened version of the token test. Cortex 1978;14:419 .

10 Stroop J. Studies of interference in serial verbal reactions. $\mathcal{F}$ Exp Psychol 1935;18:643-62.

11 Borkowski JG, Benton AL, Spreen O. Word fluency and brain damage. Neuropsychologia 1967;5:135-40.

12 Benton AL. The revised visual retention test. Iowa: The State University of Iowa Press, 1963

13 Raven JC. Guide to the standard progressive matrices. London: HK Lewis, 1958

14 Wechsler D. WAIS-R manual. New York: Psychological Corporation, 1981
15 Berg EA. A simple objective test for measuring flexibility in thinking. f Gen Psychol 1948;39:15-22.

16 Spreen O, Strauss E. A conpendium of neuropsychological tests. Oxford: Oxford University Press, 1991.

17 Wechsler D. Wechsler memory scale-revised. San Antonio: Harcourt Brace Jovanovich, 1987.

18 Bowers D, Verfaellie M, Valenstein E, et al. Impaired acquisition of temporal information in retrosplenial amnesia. Brain Cogn 1988;8:47-66.

19 Squire LR, Nadel L, Slater PC. Anterograde amnesia and memory for temporal order. Neuropsychologia 1981;8:7581 .

20 Shuren JE, Jacobs DH, Heilman KM. Diencephalic temporal order amnesia. I Neurol Neurosurg Psychiatry 1997;62: 163-8.

21 Stephanie C, Assal G, Bogousslavsky J, et al. Pure amnesia after unilateral left polar thalamic infarct: topographic and sequential neuropsychlogic and metabolic (PET) correlations. F Neurol Neurosurg Psychiatry 1994;57:27-34.

22 Deutsch JA. Chromomnemonics and amnesia. In: Squire LR, Butters N, eds. Neuropsychology of memory. New York: Guilford Press, 1984:157-64.

23 Schacter DL, Harbluk JL, MaLachlan DR. Retrieval Schacter DL, Harbluk JL, MaLachlan DR. Retrieval
without recollection: an experimental analysis of source amnesia. F of Verbal Learning and Verbal Behaviour 1984;23: 593-611.

24 Parkin JA, Hunkin NM. Impaired temporal context memory on anterograde but not retrograde tests in the absence of frontal pathology. Cortex 1993;29:267-80. 